\title{
Capacidade absortiva e orientação empreendedora dos jornais brasileiros
} Absorptive capacity and entreprise guidance of Brazilian newspapers

\author{
Marina Adriano de Andrade \\ Faculdade lelusc \\ email: mariandrade.jornal@gmail.com
}

\section{Fernando César Lenzi}

Universidade do Vale do Itajaí (Univali)

email:enzisc@gmail.com

\section{Carlos Ricardo Rossetto}

Universidade do Vale do Itajaí (Univali)

email: rossetto@univali.br

Sayonara de Fatima Teston

Universidade do Oeste de Santa Catarina (Unoesc)

email:sayonara.teston@unoesc.edu.br

\section{RESUMO}

Ambientes turbulentos exigem das firmas capacidade de aprender e aplicar o conhecimento de maneira efetiva para garantir processos empreendedores, inovações e resultado. Esta pesquisa busca avaliar a capacidade absortiva dos jornais brasileiros com a orientação empreendedora destas organizações. O objeto do estudo foi escolhido levando-se em consideração o momento de transformação deste tipo de negócio a partir das consequências que a internet trouxe para a forma como as pessoas consomem informação. Com uma abordagem quantitativa, o trabalho conclui que a capacidade absortiva das empresas estudadas influencia na orientação empreendedora das mesmas. O resultado foi obtido a partir de uma survey aplicada com os principais gestores de empresas que possuem veículos de mídia impressa no Brasil. Além de comprovar a hipótese da pesquisa por meio de uma modelagem de equação estrutural, o estudo utiliza análises estatísticas para identificar e mensurar as dimensões de OE (LUMPKIN; DESS, 1996) e ACAP (ZAHRA; GEORGE, 2002).

Palavras-Chave: Orientação Empreendedora. Capacidade Absortiva. Jornalismo Impresso.

\section{ABSTRACT}

Turbulent environments require firms to be able to learn and apply knowledge effectively to ensure entrepreneurial processes, innovations, and results. This research aims to evaluate the absorptive capacity of Brazilian newspapers with the entrepreneurial orientation of these organizations. The study object was chosen to take into account the moment of transformation of this business type from the consequences that the internet has brought to the way people consume information. With a quantitative approach, the paper concludes that the absorptive capacity of the studied companies influences their entrepreneurial orientation. The result from a survey applied to the primary managers of companies that own print media in Brazil. In addition to proving the research hypothesis through structural equation modeling, the study uses statistical analyses to identify and measure the OE (LUMPKIN; DESS, 1996) and ACAP (ZAHRA; GEORGE, 2002) dimensions.

Key-words: Entrepreneurial Orientation. Absorptive capacity. Printed journalism. 


\section{INTRODUÇÃO}

As primeiras iniciativas de jornalismo na internet foram registradas nos Estados Unidos nos anos 1980. A partir da década seguinte, grandes periódicos daquele país e de outras partes do mundo, especialmente da Europa, tornaram-se adeptos de plataformas digitais, chegando a 150 jornais online em 1995.

Entre 1997 e 1999, quando um estudo apontou para pelo menos dez mil veículos com presença na internet no mundo, a audiência digital se consolidava e a possibilidade de virtual substituir os impressos já era tema de debate em fóruns especializados na Europa (DÍAZ-NOCI, 2013). As apostas em publicidade começaram a dividir espaço com os conteúdos pagos em busca de assinantes online com maior intensidade, enquanto também se procurava, com dificuldades, um modelo sustentável para bancar as organizações produtoras de jornalismo digital (GREER; MENSING, 2006).

Enquanto isso, no Brasil, Alcadipani (2007) chamava atenção para a crise vivida por este mercado. Apesar de os resultados das pesquisas direcionarem para a necessidade destas organizações se comportarem de forma mais estratégica e inovarem sua maneira de atuação, e de parte estar trabalhando com o fim de desenvolver um modelo de negócio de sucesso, são raros os exemplos de firmas bem-sucedidas (KARIMI; WALTER, 2016; CESTINO; MATTHEWS, 2016; DEUZE, 2016).

Dados do Instituto Verificador de Comunicação (IVC) apresentados em relatório da Associação Nacional de Jornais (ANJ) em 2015 mostram que entre janeiro de 2014 e setembro do ano seguinte, o acesso às edições digitais cresceu de 427.370 para 641.776, o que significa $50 \%$ de aumento. Já as edições impressas registraram queda de $8,6 \%$, passando de 3.834 .613 para 3.505.838. A diminuição da circulação total dos jornais foi de $2,7 \%$. Apesar de jornais impressos virem perdendo espaço no quesito mercadológico, uma campanha elaborada em 2015 pela mesma ANJ defende, a partir de dados provenientes de diferentes levantamentos, que o interesse no produto notícia é cada vez maior. Segundo a associação, 92\% dos usuários de smartphones no Brasil leem notícias pelo celular e houve crescimento de $461 \%$ no acesso mobile dos principais jornais do Brasil. No mundo, são 800 milhões de adultos ( $12 \%$ da população) lendo jornais em formato digital no celular e 2,5 bilhões de pessoas consumindo jornais impressos regularmente.

Ainda que o crescimento no acesso aos jornais digitais seja animador, apenas uma migração de plataforma impressa para digital não é apontada como solução garantida para que este tipo de organização seja capaz de cobrir seus custos de produção e distribuição de conteúdo.

Em mercados turbulentos, as firmas precisam ofertar produtos ou serviços com frequência e inovar constantemente para responder a tempo as mudanças no comportamento e preferências do consumidor (JAWORSKI; KOHLI, 1993; SLATER; NARVER, 1995; ENGELEN et al., 2014). A forma como as organizações empreendem, ou seja, qual o processo empreendedor das firmas, suas práticas, ações e atividades para gerenciamento e tomada de decisões em busca de competitividade e melhor descoberta e aproveitamento de oportunidades é entendido como orientação empreendedora (OE) (LUMPKIN; DESS, 1996; SHANE; VENKATARAMAN, 2000; DESS; LUMPKIN, 2005; DE CLERCQ et al., 2013; TONDOLO et al., 2014). A OE é um caminho viável para a inovação como fator de diferenciação (FRANÇA; SARAIVA; HASHIMOTO, 2012).

Pesquisar a forma como as organizações empreendem envolve estudar também a maneira como estas identificam oportunidades de mercado e criam combinações de recursos para encontrar estas possibilidades (SCHUMPETER, 1934; KIRZNER, 1973; GUTH; GINSBERG, 1990). Neste sentido, Cohen e Levinthal (1990) introduzem o conceito de capacidade absortiva (ACAP), entendida pelos autores como um recurso, o qual se caracteriza pelo processo de aprendizagem na organização para que esta gere valor e sustente vantagem competitiva.

Diante dos desafios expostos até aqui, entende-se que o momento atual das organizações que produzem jornais impressos no Brasil exige processos sólidos e ações que levem à orientação empreendedora. Sendo assim, a pesquisa pretende analisar a relação entre a capacidade absortiva dos jornais impressos do Brasil e a orientação empreendedora destas organizações.

Covin e Lumpkin (2011) consideram estudos das relações entre OE e capacidades dinâmicas com grande potencial de valor. Para criarem oportunida- 
des empreendedoras por meio de suas atitudes, as organizações necessitam reconfigurar seus recursos e as capacidades dinâmicas são o mecanismo para tal. Engelen et al. (2014) sugerem a necessidade de examinar mais profundamente a interação entre a orientação empreendedora, a capacidade absortiva e os mercados turbulentos, especialmente em países menos desenvolvidos. Além disso, ao analisar empiricamente a relação entre orientação empreendedora e capacidade absortiva em organizações brasileiras, $o$ trabalho colabora para o enriquecimento deste campo de estudo em países em desenvolvimento, conforme necessidade salientada em trabalhos anteriores (ENGELEN et al. 2014; MARTENS, 2016).

\section{FUNDAMENTAÇÃO TEÓRICA}

A fundamentação teórica buscou clarear os conceitos centrais do estudo.

\subsection{ORIENTAÇÃO EMPREENDEDORA}

O conceito de orientação empreendedora decorre de estudos da escola canadense de estratégia, especialmente na década de 1980 e tem Danny Miller como pioneiro e um dos principais autores (FREITAS et al., 2012). O trabalho de Mintzberg (1973) sobre a tomada de decisão estratégica é apontado por ele como uma das raízes dos estudos deste constructo (COVIN; WALES, 2012). Para Miller (1983) empresa empreendedora é a que se dedica à inovação de mercado/produto, empreende empreendimentos um tanto arriscados e é a primeira a apresentar inovações proativas, batendo os concorrentes com duros golpes. Desta colocação é possível extrair as três dimensões da OE listadas pelo autor: inovatividade, assunção de risco e proatividade.

Lumpkin e Dess (1996) assumem empreendedorismo como nova entrada podendo esta ser em uma nova firma em um mercado estabelecido ou novo. Covin e Lumpkin (2011) argumentam que para que características da firma como estrutura organizacional e cultura possam realmente fazer destas organizações empreendedoras, é necessário que os atributos estejam explicitados em suas ações.
Miller (1983), seguido por Covin e Slevin (1989), trabalhavam com uma abordagem unidimensional da OE, na qual as três dimensões teriam o mesmo alto grau de contribuição na caracterização de uma organização empreendedora. Já Lumpkin e Dess (1996) adotaram uma abordagem multidimensional. Para eles, a intensidade de cada uma das dimensões não é necessariamente semelhante.

Ao revisitar o artigo de 1983, Miller (2011) relembrou elementos do estudo pioneiro que levavam para uma visão multidimensional, trabalhada após autores como Lumpkin e Dess (1996) e reconheceu as duas dimensões acrescentadas pelos autores. Para este trabalho, são consideradas as cinco dimensões sugeridas por Lumpkin e Dess (1996) a partir de uma análise multidimensional, considerando que cada uma destas, detalhadas a seguir, pode se manifestar em grau diferente de acordo com o tipo de organização:

1. Inovatividade: tendência da organização se engajar e apoiar novas ideias, experimentação e processos criativos que podem resultar em novos produtos, serviços ou processos. É também uma forma de medir a propensão da organização desenvolver novidades ou inovações, tendo a abertura para a criação como uma cultura interna (HURLEY; HULT, 1998). Investimento em inovação ou desenvolvimento, crescimento do leque ou velocidade de mudança dos serviços ou produtos oferecidos podem ser sinais para mensurar o nível de inovatividade (COVIN; SLEVIN, 1989; MILLER; FRIESEN,1982; FREITAS et al., 2012);

2. Assunção de riscos: tendência de embarcar em projetos de risco (MILLER, 1983). Pode ser vista a partir de três perspectivas: 1 . Do negócio, quando está associada à entrada em novos mercados não testados ou usando tecnologias desconhecidas; 2. Financeiros, quando há grande aporte ou comprometimento de recursos; e 3. Pessoais, que envolve a tomada de decisão do executivo apesar do risco de impacto negativo em sua carreira (LUMPKIN; DESS, 2001).

3. Proatividade: estar à frente no desenvolvimento e introdução de novos produtos e tecnologias ao invés de apenas seguir o mercado. Também se refere à ousadia dos gestores e pode 
ser mensurada a partir do envolvimento das organizações em projetos intrépidos (MILLER, 1983; FREITAS et al., 2012). Lumpkin e Dess (2001) destacam que esta dimensão é particularmente diferenciada na geração de vantagem competitiva por estimular a reação a iniciativas bem-sucedidas por parte dos competidores. Lumpkin e Dess (1996) apontam:

4. Autonomia: com relação direta com a equipe, reflete como líderes e equipes têm liberdade para apresentar um comportamento mais independente, menos centralizado, e com menor dependência da delegação de tarefas ou processos (LUMPKIN; DESS, 1996, 2001). Miller (1983) define a liberdade para agir de forma independente como crucial na dimensão de OE. A autonomia pode sofrer influência do tamanho da organização e do seu estilo gerencial (FREITAS et al., 2012).

5. Agressividade competitiva: tendência a responder com vigor a concorrência e suas ameaças em busca de vantagem competitiva para ter melhores resultados. Está ligada à intensidade com que a organização luta por sua sobrevivência, podendo abrir mão de lucratividade para conquistar market share, por exemplo, sempre com técnicas e pesquisas para que os riscos sejam minimizados (LUMPKIN; DESS, 2001). Esta dimensão pode ocorrer concomitante com as demais (FREITAS et al., 2012).

A partir das dimensões, a OE captura uma postura estratégica da firma para a busca de novas oportunidades de crescimento e renovação organizacional, o que favorece as atividades empreendedoras, as decisões estratégicas e as filosofias gerenciais (COVIN; WALES, 2012; WALES; PARIDA; PATEL, 2012). Camozzato et al. (2017) corroboram já que avaliaram as relações da orientação empreendedora, medida pela assunção de risco e agressividade competitiva, a formação e conhecimento dos colaboradores, e a autoeficácia empreendedora do gestor com a sua satisfação no desempenho de empresas incubadas. Os autores concluíram que a assunção de risco e a autoeficácia possuem vínculo positivo e significante com a satisfação no desempenho, e que os preditores possibilitam estimar o desempenho, sendo a escala total da autoeficácia a que tem maior poder preditivo, bem como, que a definição do principal objetivo do negócio e a construção de um ambiente de inovação, apresentam potencial preditor semelhante ao da escala total (CAMOZZATO et al., 2017). Lizote et al. (2020) também investigaram orientação empreendedora em conjunto com outras variáveis e concluíram que conhecer sobre a forma predominante de orientação por parte de gestores hoteleiros auxilia no processo de tomada de decisões.

\subsection{CAPACIDADE ABSORTIVA}

Ao conceituar capacidades dinâmicas (CD), Teece, Pisano e Shuen (1997) focam na questão do processo da firma na hora de criar e renovar competências, sendo este derivado tanto de experiências passadas da organização quanto pelo efeito do dinamismo do ambiente. Para Eisenhardt e Martin (2000), capacidades dinâmicas consistem em rotinas identificáveis e específicas. Estas são sustentadas em um tripé composto por processos (rotinas, práticas e aprendizado); posições (estrutura, ativos, relações externas); e trajetória (passado de decisões) (TEECE; PISANO; SHUEN, 1997; MEIRELLES; CAMARGO, 2014). As CD refletem o aspecto empreendedor da administração, especialmente quando se considera uma visão schumpeteriana na qual a competição tem a inovação em sua base (TEECE; PISANO; SHUEN, 1997; TEECE, 2003).

Wang e Ahmed (2007) listaram, a partir de achados empíricos, as capacidades de adaptação, absorção e inovação como sendo os três principais aspectos em comum entre as capacidades das organizações: adaptativa, inovativa, absortiva.

A capacidade absortiva (ACAP) ganha destaque nesta pesquisa por ser necessária especialmente em ambientes turbulentos e é vista como um dos principais fatores determinantes para o empreendedorismo corporativo por melhorar o reconhecimento e exploração de novas oportunidades por meio da construção de novas habilidades, além de contribuir para diminuir a rigidez cognitiva entre os gerentes seniores de uma empresa (SCIASCIA et al., 2014). É a que mais se relaciona com o fato de que em mercados turbulentos, mudanças no consumidor e nas suas preferências ocorrem de forma rápida. Quando o 
mercado é dinâmico, a organização precisa melhorar frequentemente suas ofertas e inovar para responder em tempo estas alterações (JAWORSKI; KOHLI, 1993; SLATER; NARVER, 1995).

Lane et al. (2006) entendem a capacidade absortiva como multinível (organizacional e individual e destacam que ela depende de processos e rotinas dentro da organização que permitem que esta compartilhe, comunique e transfira a aprendizagem de nível individual para o nível organizacional. Assim como Cohen e Levinthal (1990), dividem a capacidade absortiva em três categorias de aprendizagem: a) exploratória: reconhecer e entender novos conhecimentos externos. O conhecimento prévio da empresa é uma função de modelos mentais individuais que influenciam a avaliação do valor dos novos conhecimentos; b) transformadora: assimilar valiosos conhecimentos externos a partir de vários processos que afetam a forma como o conhecimento recém-adquirido e combinado com o existente na organização. No nível organizacional, os processos de gerenciamento de conhecimento afetam a forma como esse conhecimento é compartilhado e transferido; e, c) exploradora: como o aprendizado é usado para aplicar o conhecimento externo assimilado.

ACAP é geralmente compreendida como um processo com passos que estão estreitamente ligados (ZAHRA; GEORGE, 2002; ENGELEN et al., 2014). O processo de reconhecer, adquirir, assimilar e aplicar o conhecimento, explorando-o de forma que contribua positivamente para o resultado reflete o conceito de capacidade e ganha importância em ambientes de rápidas mudanças, nos quais é necessário aprender fazendo (COHEN; LEVINTHAL, 1990; WALES; PARIDA; PATEL, 2013).

Cassol (2014), com base no trabalho de Zahra e George (2002) e no de outros autores, divide os diferentes níveis de interação da capacidade absortiva em: (1) individual, no qual o foco é o funcionário; (2) grupal, ou seja, em times, divisões de negócios e filiais; (3) organizacional; e (4) interorganizacional, quando envolve diferentes níveis de interações, como alianças estratégicas e sistemas nacionais de inovação.

No âmbito organizacional, para exercer a capacidade absortiva, a empresa depende de sua experiência interna, do conhecimento especializado e de processos apropriados, que lhe permitam identificar e utilizar o significado das ideias externas e oportunidades de inovações (PADILHA et al., 2016). Zahra e George (2002) detalham a ACAP como uma construção multidimensional que incide em diversos momentos em diferentes processos e rotinas e apontam para a existência de dois subconjuntos de capacidade de absorção: 1) Potencial (PAC), que possibilita a receptividade da empresa ao conhecimento externo e é formada pela aquisição e assimilação; 2) Realizada (RAC), que reflete a capacidade de uma organização de alavancar o conhecimento absorvido (explorar) e transformá-lo em inovação. A capacidade de transformação desse conhecimento é uma evolução da obra de Cohen e Levinthal (1990) que consideravam três dimensões para ACAP.

Zahra e George (2002) ressaltam que as quatro capacidades dos dois subconjuntos são complementares. E que estes atributos dão base para que as organizações desenvolvam tipos de vantagem competitiva. Desta forma, é possível definir cada uma das quatro dimensões da ACAP da seguinte forma: a) Aquisição: identificar e adquirir conhecimento gerado externamente considerado relevante para sua operação. Os esforços nas rotinas para adquirir conhecimento têm três atributos que podem influenciar a ACAP: intensidade, velocidade e direção. Os dois primeiros podem determinar a qualidade de aquisição de conhecimento por parte da firma. Já a direção implica em ter diferentes áreas de expertise para importar conhecimento de forma adequada; b) Assimilação: rotinas e processos que permitem que a organização analise, processe, interprete e entenda a informação obtida de fontes externas; c) Transformação: capacidade de desenvolver e refinar rotinas que combinem o conhecimento existente com o que acabou de ser adquirido e assimilado. Combinar informações aparentemente incongruentes para formar novo conhecimento; d) Exploração: rotinas que permitem refinar, ampliar e alavancar as competências existentes ou criar novas, incorporando conhecimento adquirido, assimilado e transformado em operações. As empresas podem ser capazes de explorar o conhecimento de forma acidental, sem rotinas sistemáticas. No entanto, a presença dessas rotinas fornece mecanismos estruturais, sistêmicos e processuais que permitem a exploração do conhecimento por longos períodos.

Rossetto et al. (2019) afirmam que as pressões competitivas para a maioria das empresas exigem 
estratégias de gestão inovadoras, eficientes e eficazes para sobreviver e prosperar no longo prazo. Nesse sentido, a capacidade absortiva tem contribuído para a compreensão de diversos fenômenos organizacionais, principalmente aqueles relacionados aos processos de gestão do conhecimento, aprendizagem, inovação e desempenho. Cappellari et al. $(2019$, p. 2) corroboram, e demonstram que organizações desenvolvem capacidade absortiva por meio de comportamentos e habilidades, por meio de rotinas e processos, e ainda mediante mecanismos de aprendizagem e governança do conhecimento. Padilha et al. (2020) acrescentam que a capacidade absortiva favorece inclusive a adoção de práticas de sustentabilidade por parte das organizações.

\subsection{ORIENTAÇÃO EMPREENDEDORA E CAPACIDADE ABSORTIVA}

O constructo da orientação empreendedora e o constructo da capacidade absortiva são fundamentais para atender ao objetivo da pesquisa. Wales, Parida e Patel (2013), ao pesquisarem 285 empresas suecas de tecnologia de pequeno e médio porte, constataram que quanto maior a $\mathrm{OE}$, maior a ACAP pode ser alcançada antes dos retornos financeiros diminuírem. Os autores adotaram a OE como moderadora da relação entre capacidade absortiva e desempenho financeiro das firmas. Com maior OE, as empresas podem identificar tendências e oportunidades para alavancar seus recursos baseados no conhecimento antes de seus concorrentes e assumir os riscos necessários para ser pioneiro em novas ofertas em mercados potenciais.

Díaz, Guerrero e Peña (2015) veem na orientação empreendedora e na capacidade absortiva duas características internas imprescindíveis para organizações inovadoras, e consideram os constructos características das firmas capazes de crias novas empresas spin-off (a partir de uma já existente). Os autores usam a OE e a ACAP como variáveis explicativas capazes de ajudar a identificar quais empresas são mais inovadoras e têm produtividade superior.

Já Silva França e Lima Rua (2016) optaram por pesquisar a influência da orientação empreendedora e da capacidade absortiva nas exportações de empresas de calçado de Portugal devido à crise enfrentada pelo país e pelo setor. Os autores justificam a escolha apontando a orientação empreendedora e a capacidade absortiva como determinantes e afirmam que estas contribuem para a formulação estratégica de políticas econômicas e gestão de negócios, visando o aumento do desempenho dos negócios nos mercados externos, agregando valor ao atual contexto de mudança (SILVA FRANÇA; LIMA RUA, 2016).

Van Doorn, Heyden e Volberda (2017) consideram que em ambientes turbulentos, a possibilidade de capitalizar uma oportunidade empreendedora é muito menor, o que faz da competência do time de alta gestão para assimilar informações do ambiente externo e fazer destas relevantes no processo de tomada de decisão, uma aliada para o sucesso no aproveitamento dessas possibilidades.

Figura 1 Modelo Teórico da Pesquisa

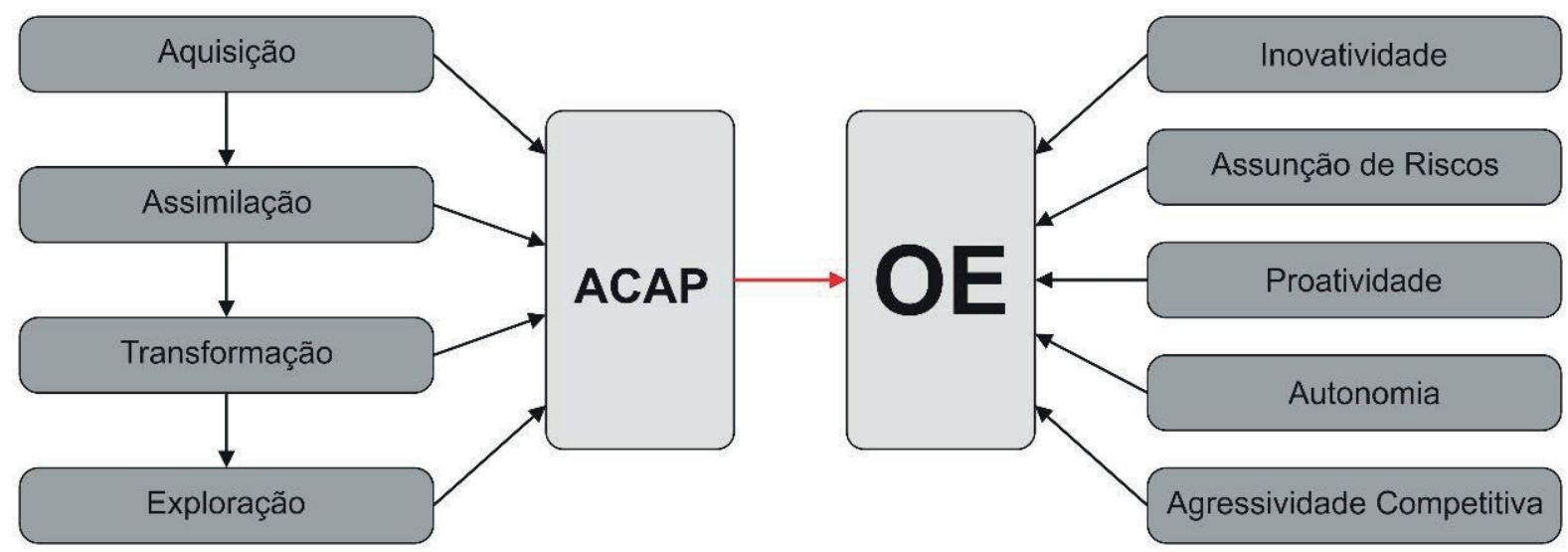

Fonte: elaborada pelos autores (2019). 
Hernandez-Perlines (2018), ao estudar empresas familiares, constatou que o desempenho internacional destas é determinado, em grande parte, pela orientação empreendedora. Além disso, este efeito é reforçado pela capacidade de absorção, exercendo um papel moderador positivo. Sbissa et al. (2018) confirmaram que a capacidade absortiva influencia positivamente na orientação empreendedora de hotéis catarinenses.

Apresentada a revisão teórica sobre orientação e capacidade absortiva, apresenta-se a seguir o modelo teórico desta pesquisa a partir de figura que representa a hipótese testada neste estudo: a capacidade absortiva influencia a orientação empreendedora.

\section{PROCEDIMENTOS METODOLÓGICOS}

A pesquisa é quantitativa, descritiva e correlacional. O universo pesquisado neste trabalho abrange organizações que produzem jornais impressos no Brasil. O primeiro passo foi verificar quais instituições ou associações reúnem os principais jornais brasileiros. Os primeiros contatos realizados foram com a Associação Nacional de Jornais (ANJ) e com o Instituto Verificador de Comunicação (IVC), que faz auditoria de veículos de todo o país.

Juntas, desconsiderando membros em comum, as instituições somam 138 jornais e elas representam 109 organizações. Destas, 45 organizações, que somam 63 jornais impressos, responderam esta pesquisa. Considerando-se um nível de confiança de $90 \%$ e um erro amostral de $10 \%$, este número já representaria a amostra necessária para o levantamento. Além das organizações que fazem parte do IVC e ANJ outras empresas jornalísticas responderam ao questionário, fazendo com que este estudo some um total de 62 participantes.

$\mathrm{O}$ instrumento de pesquisa foi dividido em três blocos e soma 57 questionamentos. A primeira parte busca a caracterização do respondente e principalmente da empresa representada por este, visto que o estudo se dá no âmbito organizacional. São onze perguntas, parte com respostas abertas e parte de múltipla escolha. Já os questionamentos do segundo bloco (sobre orientação empreendedora) e do terceiro (sobre capacidade absortiva) seguem a escala tipo Likert, de sete pontos, considerando "1" para de "discordo totalmente" e "7" para "concordo totalmente".

O bloco sobre Orientação Empreendedora foi construído com base nas cinco dimensões propostas por Lumpkin e Dess (1996). Por isso foi utilizada a escala já validada desenvolvida por Martens et al. (2015), adaptada por Sbissa (2017). A linguagem foi mudada para se enquadrar ao segmento estudado. As assertivas do bloco sobre ACAP tem como base o trabalho de Tenconi (2015). O mesmo instrumento foi utilizado por Sbissa (2017).

Para a coleta de dados o instrumento foi inserido na plataforma Google Forms e o link com as perguntas enviado por e-mail para a lista construída especificamente para esta pesquisa. Em paralelo, foram feitos contatos por telefone e via WhatsApp com uma rede de relacionamento do setor para garantir maior aderência. Foram feitas ainda três rodadas de telefonemas. O trabalho foi desenvolvido entre os dias 5 e 22 de junho de 2018. Os 62 respondentes representam 91 jornais brasileiros.

Neste estudo foram utilizadas técnicas básicas de estatística descritiva, sendo estas análises fatoriais dos tipos exploratória e confirmatória e modelagem de equações estruturais.

Efetuou-se a média entre as respostas repetidas de uma mesma empresa, situação ocorrida em sete casos, visto que este estudo se dá no âmbito organizacional. Posteriormente, um novo arquivo foi gerado com as 62 respostas válidas para que estas fossem submetidas a técnicas básicas de estatística descritiva. Todos os respondentes válidos estavam completos em relação às perguntas sobre os construtos e suas dimensões. Avaliou-se a normalidade da distribuição por meio do cálculo da assimetria, que deve estar entre -2 até +2 ; e da curtose, que deve estar em um intervalo de entre -7 e +7 (FINNEY; DISTEFANO, 2013; HAIR et al., 2009).

$\mathrm{Na}$ sequência, foi realizada uma Análise Fatorial Exploratória (AFE) para entender o tipo de distribuição das variáveis e a relação da linearidade destas, além das medidas aleatórias de erro (HAIR et al., 2009). Calculou-se previamente o coeficiente alfa de Cronbach para cada construto e a correlação média inter-itens e do item total (CHURCHILL Jr., 
1979). A viabilidade da análise fatorial foi testada com KMO (Kaiser, Olkin e Meyer) e Bartlett.

As restrições empregadas na AFE incluíram a retirada por fatores principais, que não requer multinormalidade, seguindo o preceito de Kaiser a partir de matrizes de correlações. Também foram consideradas apenas cargas fatoriais maiores ou iguais a 0,60 em módulo e a comunalidade maior ou igual que 0,36 . Já a variância retirada pelo fator em caso de unidimensionalidade deve ser maior ou igual que 50\%. Ainda segundo Hair Jr. et al. (2009), na matriz de correlações, o padrão de correlação entre as variáveis deve exibir a maior parte dos coeficientes com valor superior a 0,30 .

O teste de hipóteses em relação a estrutura do conjunto de dados foi feito por meio de análise fatorial confirmatória (AFC). Garcia e Martinez (2003) argumentam que este procedimento corrige deficiências da AFE, garantindo mais certeza das hipóteses a serem confrontadas a partir de modelos que expliquem o inter-relacionamento existente na estrutura de um questionário.

A partir destas análises foram desconsideradas duas assertivas do construto OE: AR-4, relativa à assunção de risco, e AC-4, que compunha a dimensão agressividade competitiva. Seguindo o que é sugerido por Hair Jr. et al. (2009), foi aplicada análise fatorial confirmatória (AFC). A AFC confirmou os modelos utilizados, tanto por construto quanto geral, este último fundamentado na revisão teórica deste trabalho. Utilizou-se como restrição a obrigatoriedade de os indicadores apresentarem um coeficiente padronizado entre o indicador e o construto avaliado de, pelo menos, 0,50 .

\section{APRESENTAÇÃO DE RESULTADOS}

A apresentação dos resultados é constituída pela caracterização dos participantes, análise descritiva das variáveis, análise fatorial exploratória, análise fatorial confirmatória, relacionamento entre os constructos e discussão.

\subsection{CARACTERIZAÇÃO DOS PARTICIPANTES}

A maior parte dos entrevistados se identificou com o cargo de diretor (42\%) e são de organizações localizada nas regiões Sul (37\%) e Sudeste (37\%) do país. Quanto a área de abrangência dos jornais, apenas $8 \%$ das organizações pesquisadas trabalham com abrangência nacional, enquanto $58 \%$ possuem abrangência estadual e 34\% local/regional. Quanto à média de exemplares impressos e distribuídos a cada edição, $11 \%$ possuem até 5 mil exemplares, $32 \%$ entre 5.001 e 10.000 exemplares, $23 \%$ entre $10.001 \mathrm{e}$ $20.000,23 \%$ entre 20.001 e 60.000 , e $11 \%$ acima de 60.001 exemplares.

Entre os respondentes, predominam empresas com circulação de entre 5.001 mil e 60 mil exemplares por dia, com destaque para organizações com circulação diária de entre 5.001 e 10.00 mil exemplares, ou seja, empresas com abrangência provavelmente regional/local. Sobre a principal estratégia de vendas adotada, 3\% trabalham com comercialização por assinatura e venda avulsa, $78 \%$ com assinatura e 19\% com venda avulsa. Além disso, $99 \%$ das organizações possuem pelo menos mais negócios além do jornal impresso, porém para $69 \%$ das respondentes o veículo impresso ainda é a principal fonte de receita. E, mais da metade dos participantes (55\%) tem um jornal impresso há mais de 50 anos.

\subsection{ANÁLISE DESCRITIVA DAS VARIÁVEIS}

Após a verificação da quase normalidade (FINNEY; DISTEFANO, 2013) dos itens dos constructos Orientação Empreendedora (OE) e Capacidade Absortiva (ACAP), foram calculados o coeficiente alfa de Cronbach para cada um dos dois construtos, além da correlação média inter-itens e do item com o total (Churchill Jr., 1979). Também foram utilizados os testes de Kaiser, Olkin e Meyer (KMO), o de Bartlett e a comunalidade. Todos com o objetivo de confirmar a factibilidade de empregar a análise fatorial. Primeiro, os cálculos foram realizados tendo em vista cada uma das cinco dimensões de OE e quatro de ACAP. Os resultados são apresentados na Tabela 1.

Os valores encontrados permitem o emprego da análise fatorial. 
Tabela 1 Indicadores de factibilidade de fazer uma Análise Fatorial Exploratória

\begin{tabular}{|c|c|c|c|c|c|c|}
\hline \multirow{2}{*}{ Constructo } & \multirow{2}{*}{ Dimensões } & \multirow{2}{*}{ a de Cronbach } & \multicolumn{2}{|c|}{ Correlações } & \multirow{2}{*}{ KMO } & \multirow{2}{*}{ Comunalidade } \\
\hline & & & Inter-itens & Item-total & & \\
\hline \multirow{5}{*}{$\mathrm{OE}$} & Inovatividade & 0,685 & $>0,367$ & $>0,306$ & 0,658 & $>0,312$ \\
\hline & Assunção de risco & 0,781 & $>0,484$ & $>0,442$ & 0,579 & $>0,409$ \\
\hline & Proatividade & 0,657 & $>0,337$ & $>0,300$ & 0,566 & $>0,327$ \\
\hline & Autonomia & 0,725 & $>0,400$ & $>0,407$ & 0,732 & $>0,407$ \\
\hline & Agres. Competitiva & 0,705 & $>0,381$ & $>0,367$ & 0,678 & $>0,357$ \\
\hline \multirow{4}{*}{ ACAP } & Aquisição & 0,864 & $>0,522$ & $>0,610$ & 0,764 & $>0,523$ \\
\hline & Assimilação & 0,894 & $>0,634$ & $>0,685$ & 0,829 & $>0,638$ \\
\hline & Transformação & 0,936 & $>0,690$ & $>0,715$ & 0,884 & $>0,616$ \\
\hline & Aplicação & 0,920 & $>0,708$ & $>0,711$ & 0,820 & $>0,656$ \\
\hline
\end{tabular}

Fonte: elaborada pelos autores (2019).

Tabela 2 Itens selecionados para as dimensões da Orientação Empreendedora

\begin{tabular}{c|c|c|c|c|c|c|c|c|c}
\hline \multicolumn{2}{c|}{ Inovatividade } & \multicolumn{2}{c|}{ Assunção de Risco } & \multicolumn{2}{c|}{ Proatividade } & \multicolumn{2}{c|}{ Autonomia } & \multicolumn{2}{c}{ Agres. Competitiva } \\
\hline Variable & Fator $\mathbf{1}$ & Variable & Fator $\mathbf{1}$ & Variable & Fator 1 & Variable & Fator 1 & Variable & Fator $\mathbf{1}$ \\
\hline IN-1 & $-0,851$ & AR-1 & $-0,852$ & PR-1 & $-0,522$ & AU-1 & $-0,734$ & AC-1 & $-0,851$ \\
\hline IN-2 & $-0,558$ & AR-2 & $-0,640$ & PR-2 & $-0,770$ & AU-2 & $-0,638$ & AC-2 & $-0,598$ \\
\hline IN-3 & $-0,573$ & AR-3 & $-0,779$ & PR-3 & $-0,616$ & AU-3 & $-0,802$ & AC-3 & $-0,769$ \\
\hline IN-4 & $-0,867$ & AR-5 & $-0,833$ & PR-4 & $-0,880$ & AU-4 & $-0,785$ & AC-5 & $-0,689$ \\
\hline Var. Expl. & 2,116 & Var. Expl. & 2,434 & Var. Expl. & 2,018 & Var. Expl. & 2,204 & Var. Expl. & 2,148 \\
\hline$\%$ Var. & 52,90 & $\%$ Var. & 60,86 & $\%$ Var. & 50,45 & $\%$ Var. & 55,11 & $\%$ Var. & 53,70 \\
\hline
\end{tabular}

Fonte: elaborada pelos autores (2019).

Tabela 3 Itens selecionados para as dimensões da ACAP

\begin{tabular}{|c|c|c|c|c|c|c|c|}
\hline \multicolumn{2}{|c|}{ Aquisição } & \multirow{2}{*}{\multicolumn{2}{|c|}{ Assimilação }} & \multicolumn{2}{|c|}{ Transformaçao } & \multirow{2}{*}{\multicolumn{2}{|c|}{ Aplicação }} \\
\hline AQ-1 & $-0,780$ & & & TR-1 & $-0,872$ & & \\
\hline$A Q-2$ & $-0,723$ & AS-1 & $-0,876$ & TR-2 & $-0,807$ & AP-1 & $-0,863$ \\
\hline$A Q-3$ & $-0,738$ & AS-2 & $-0,868$ & TR-3 & $-0,918$ & AP-2 & $-0,810$ \\
\hline$A Q-4$ & $-0,829$ & AS-3 & $-0,836$ & TR-4 & $-0,900$ & AP-3 & $-0,901$ \\
\hline$A Q-5$ & $-0,760$ & AS-4 & $-0,814$ & TR-5 & $-0,817$ & AP-4 & $-0,925$ \\
\hline$A Q-6$ & $-0,799$ & AS-5 & $-0,799$ & TR-6 & $-0,853$ & AP-5 & $-0,858$ \\
\hline Var. Exp. & 3,580 & Var. Exp. & 3,519 & TR-7 & $-0,785$ & Var. Exp. & 3,805 \\
\hline$\%$ Var. & 59,66 & $\%$ Var. & 70,39 & Var. Exp. & 5,076 & \% Var. & 76,11 \\
\hline & & & & $\%$ Var. & 72,52 & & \\
\hline
\end{tabular}

Fonte: elaborada pelos autores (2019). 


\subsection{ANÁLISE FATORIAL EXPLORATÓRIA (AFE)}

Na Tabela 2 se exibem os itens selecionados para a Orientação Empreendedora e na Tabela 3 os da ACAP.

Somente para as dimensões Assunção de Risco e Agressividade Competitiva foi necessário excluir itens por não terem atingindo as cargas fatoriais esperadas.

No caso da ACAP todos os itens das quatro dimensões foram mantidos, por suas cargas fatoriais sempre superarem 0,7. Levando em consideração esses resultados, optou-se por consolidar cada dimensão em uma única variável e, para tanto, foram feitos os somatórios das pontuações dadas a cada item. Consequentemente, o construto Orientação Empreendedora ficou expresso por cinco variáveis e a Capacidade Absortiva por quatro, nos dois casos, cada uma delas se referem às suas dimensões. A seguir, calcularam-se os indicadores usuais para definir se é possível realizar a análise fatorial, conforme Tabela 4 .

Tabela 4 Indicadores de factibilidade para a Análise Fatorial Exploratória com as somas

\begin{tabular}{c|c|c|c|c|c}
\hline \multirow{2}{*}{ Constructo } & \multirow{2}{*}{ a de Cronbach } & \multicolumn{2}{|c|}{ Correlação } & \multirow{2}{*}{ KMo } \\
\cline { 3 - 5 } & & Inter-itens & Item-total & \\
\hline OE & 0,832 & $>0,506$ & $>0,532$ & 0,781 & $>0,461$ \\
\hline ACAP & 0,885 & $>0,674$ & $>0,615$ & 0,795 \\
\hline
\end{tabular}

Fonte: elaborada pelos autores (2019).

Os valores indicam que os dados levantados permitem o emprego da análise fatorial confirmatória.

\subsection{ANÁLISE FATORIAL CONFIRMATÓRIA (AFC)}

A análise fatorial confirmatória demonstra que todas as dimensões da orientação empreendedora e da capacidade absortiva refletem aos respectivos construtos. De acordo com o modelo utilizado, a OE é formada por cinco dimensões: inovatividade, assunção de riscos, proatividade, autonomia e agressividade competitiva, mensuradas por 22 itens neste estudo. Após os procedimentos efetuados na AFE, retiveram-se 20 dos itens. Posteriormente, foram somadas as pontuações dadas pelos respondentes àqueles itens de cada dimensão. Desta forma, obteve-se uma única variável que representa a dimensão e assim poder fazer a AFC. Os indicadores de ajustamento estão de acordo com os valores sugeridos por Hair Jr. et al. (2009).
Figura 2 Análise confirmatória do construto Orientação Empreendedora

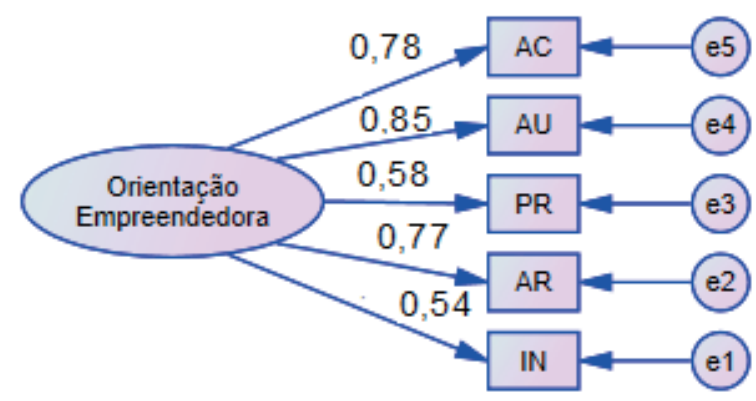

Fonte: elaborada pelos autores (2019).

A capacidade absortiva (ACAP), é formada por quatro dimensões: aquisição, assimilação, transformação e aplicação. $\mathrm{O}$ instrumento empregado para levantar os dados com os respondentes sobre este construto é composto por 23 itens, sendo todos eles retidos na AFE. Da mesma forma como foi feito com a orientação empreendedora, realizaram-se os somatórios das pontuações dos itens de cada dimensão para ter uma única variável capaz de representar a dimensão. O resultado da AFC se apresenta na Figura 3. Os indicadores de ajustamento estão de acordo com os valores sugeridos por Hair Jr. et al. (2009). 
Figura 3 Análise confirmatória do construto Capacidade Absortiva

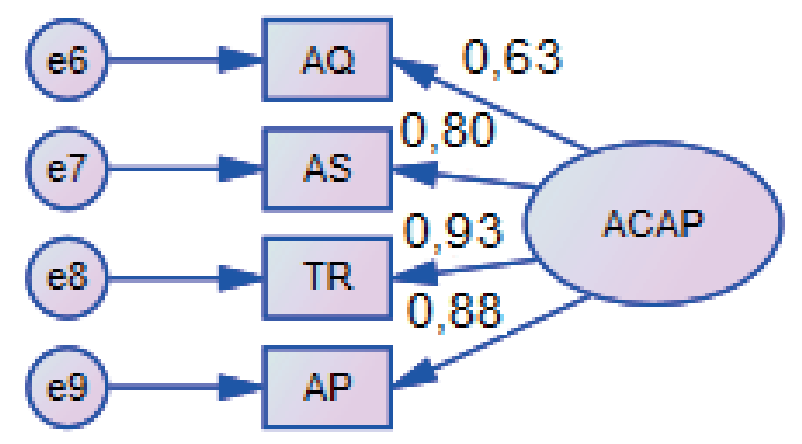

Fonte: elaborada pelos autores (2019).

\subsection{RELACIONAMENTO ENTRE OS CONSTRUTOS}

Dois procedimentos foram realizados para analisar os possíveis relacionamentos entre a orientação empreendedora e a capacidade absortiva. Um calculando as correlações de Pearson entre os somatórios das dimensões de ambos os construtos e o somatório total da orientação empreendedora e da capacidade absortiva; e o outro empregando a modelagem de equações estruturais com base nas estruturas de covariâncias.
Antes dos cálculos, avaliou-se a normalidade usando o teste de Kolmogorov-Smirnov. Todos os somatórios das dimensões da orientação empreendedora e o somatório total foram normais, com 95\% de confiança. Entretanto, no caso da capacidade absortiva não se obteve essa condição. Considerando tal fato, procurou-se avaliar os desvios da normalidade usando a assimetria e curtose. Os valores desses descritores se encontram muito aquém dos valores propostos por Finney e Distefano (2013), pelo que é aceitável calcular as correlações entre essas variáveis.

A Tabela 5 apresenta que existem correlações significativas entre todas as dimensões de ambos os construtos e ainda entre seus somatórios totais.

$\mathrm{O}$ resultado alcançado demonstra que existe um relacionamento positivo e significativo $(r=0,764$; $p=0,000)$ entre a capacidade absortiva e a orientação empreendedora. Também foi realizada modelagem de equações estruturais para avaliar a relação entre os constructos. Após criar o modelo e selecionar a base de dados, gerou-se o resultado exibido na Figura 04, onde se pode constatar que o valor padronizado da relação, isto é, em termo de correlação, chega a 0,84 , sendo muito significativo $(p<0,001)$.

Tabela 5 Correlações de Pearson entre os construtos

\begin{tabular}{|c|c|c|c|c|c|}
\hline \multirow{2}{*}{$\begin{array}{c}\mathrm{N}=62 \\
\text { Variáveis }\end{array}$} & \multicolumn{5}{|c|}{ Correlações entre OE e ACAP } \\
\hline & s-AQ & s-AS & s-TR & s-AP & s-ACAP \\
\hline \multirow{2}{*}{$s-1 N$} & 0,523 & 0,477 & 0,465 & 0,413 & 0,543 \\
\hline & $p=0,000$ & $p=0,000$ & $p=0,000$ & $p=0,001$ & $p=0,000$ \\
\hline \multirow{2}{*}{$s-A R$} & 0,430 & 0,477 & 0,504 & 0,454 & 0,541 \\
\hline & $p=0,000$ & $p=0,000$ & $p=0,000$ & $p=0,000$ & $p=0,000$ \\
\hline \multirow{2}{*}{$s-P R$} & 0,374 & 0,587 & 0,524 & 0,589 & 0,601 \\
\hline & $p=0,003$ & $p=0,000$ & $p=0,000$ & $p=0,000$ & $p=0,000$ \\
\hline \multirow{2}{*}{ s-AU } & 0,440 & 0,718 & 0,645 & 0,607 & 0,703 \\
\hline & $p=0,000$ & $p=0,000$ & $p=0,000$ & $p=0,000$ & $p=0,000$ \\
\hline \multirow{2}{*}{$s-A C$} & 0,488 & 0,605 & 0,475 & 0,438 & 0,583 \\
\hline & $p=0,000$ & $p=0,000$ & $p=0,000$ & $p=0,000$ & $p=0,000$ \\
\hline \multirow{2}{*}{$\mathrm{s}-\mathrm{OE}$} & 0,581 & 0,735 & 0,672 & 0,642 & 0,764 \\
\hline & $p=0,000$ & $p=0,000$ & $p=0,000$ & $p=0,000$ & $p=0,000$ \\
\hline
\end{tabular}

Fonte: elaborada pelos autores (2019). 


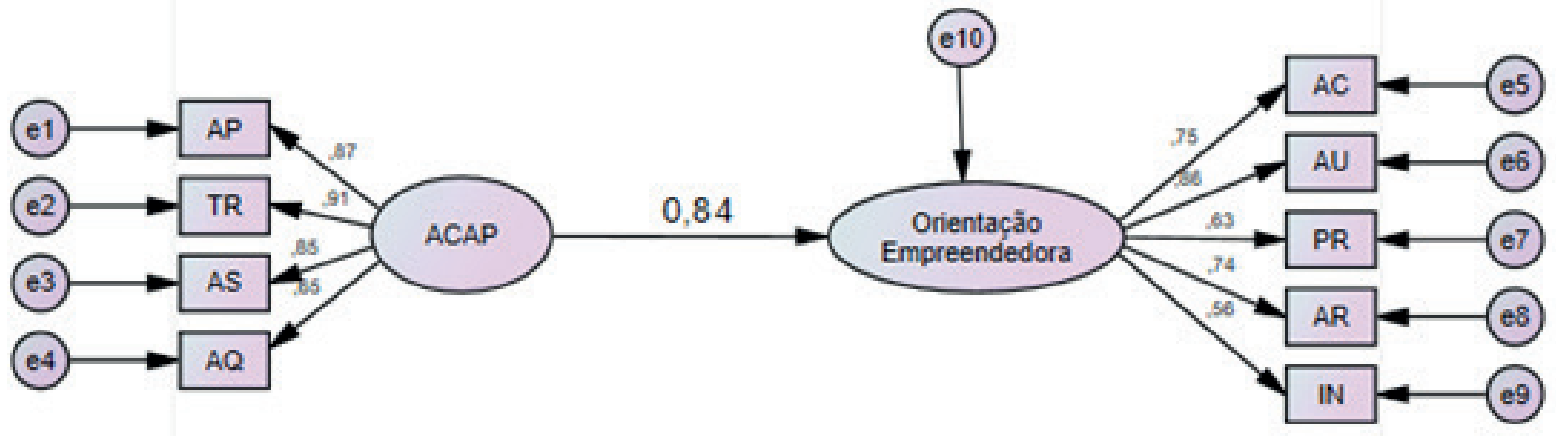

Fonte: elaborada pelos autores (2019)

O resultado obtido com a modelagem foi ainda mais significativo do que o verificado usando as correlações lineares entre as variáveis, o que ratifica que a capacidade absortiva influencia a orientação empreendedora. Os indicadores do ajustamento do modelo, apresentados na Tabela 6 , confirmam que o modelo é aceitável.

Tabela 6 Indicadores de ajustamento do modelo estrutural

\begin{tabular}{c|c|c|c}
\hline Indicador & $\begin{array}{c}\text { Valor } \\
\text { Encontrado }\end{array}$ & $\begin{array}{c}\text { Valor } \\
\text { Desejável }\end{array}$ & Interpretação \\
\hline$\left(\mathrm{X}^{2}\right) / \mathrm{gl}$ & 1,746 & $<5$ & Ótimo \\
\hline $\mathrm{CFI}$ & 0,940 & $>0,9$ & Ótimo \\
\hline $\mathrm{GFI}$ & 0,859 & $>0,9$ & Bom \\
\hline $\mathrm{NFI}$ & 0,873 & $>0,9$ & Bom \\
\hline $\mathrm{NNFI}$ & 0,916 & $>0,9$ & Ótimo \\
\hline RMSEA & 0,111 & $<0,08$ & Razoável \\
\hline
\end{tabular}

Fonte: elaborada pelos autores (2019).

A hipótese a ser testada implica no relacionamento entre os construtos capacidade absortiva e orientação empreendedora e foi suportada conforme é demonstrado pelo coeficiente significativo de correlação de 0,84 . Sendo assim, é possível afirmar que o estudo comprova que a capacidade absortiva influencia na orientação empreendedora dos jornais brasileiros.

\subsection{DISCUSSÃO}

Com as mudanças no comportamento do consumidor de notícias a partir da popularização da internet, estas firmas viram suas estratégias e processos deixarem de dar os mesmos resultados. Sentiram o impacto do aumento e da mudança da concorrência, que agora não precisa mais de jornaleiros para chegar até o público, mas sim de um simples smartphone. Diante deste cenário, estas organizações, bem como as de outros setores que também enfrentam período de turbulência, precisam encontrar alternativas inovadoras de forma rápida e eficiente, a partir de processos empreendedores. Para isso, cabe a estas terem capacidade de aprender em tempo real com o ambiente externo, onde as mudanças acontecem.

Para isso, procurou-se validar a hipótese de que ACAP influencia positivamente na $\mathrm{OE}$ das organizações pesquisadas. É importante ressaltar que a análise fatorial confirmatória feita previamente neste trabalho demonstra que todas as cinco dimensões da orientação empreendedora, sugeridas por Lumpkin e Dess (1996), bem como as quatro dimensões da ACAP, segundo Zahra e George (2002), refletem-se em cada um dos construtos. Estas dimensões se apresentam em diferentes níveis, o que caracteriza um modelo multidimensional. Isto significa que estas organizações devem atacar todas as frentes relacionadas aos construtos, avaliar processos relativos às diferentes dimensões.

Entre as cinco dimensões de OE, a proatividade se destaca na visão dos respondentes, totalizando $71,95 \%$ do total possível. Esta dimensão tem relação com a geração de vantagem competitiva por apontar 
para a tendência de a organização estar à frente no desenvolvimento e introdução de novos produtos e tecnologias. A proatividade pode ser relacionada com a capacidade absortiva da firma a partir da visão trazida pelos autores trabalhados na fundamentação teórica deste estudo de que esta dimensão implica em monitorar o mercado e antecipar necessidades, ou seja, adquirir o conhecimento. Mas também se manifesta a partir da prática, ou seja, de os gestores terem ousadia e pioneirismo para aproveitar oportunidades e gerar soluções condizentes, o que se reflete nas dimensões de assimilar, transformar e aplicar o conhecimento.

A dimensão inovatividade $(63,13 \%)$ que consiste na tendência em apoiar novas ideias e experimentar; autonomia (61\%) que reflete a aptidão da organização em dar liberdade para um comportamento mais independente para a equipe; e assunção de risco $(60,25 \%)$ que está ligada à disposição de a organização embarcar em projetos ousados e decidir sobre rumos estratégicos, também foram percebidas pelos gestores nas organizações onde atuam em grau significativo. Camozzato et al. (2017) já haviam ressaltado a importância da orientação empreendedora no desempenho de empresas incubadas, apontado principalmente para a importância da definição do principal objetivo do negócio e da construção de um ambiente de inovação.

O mesmo ocorre em relação à agressividade competitiva, que obteve $56,34 \%$ do total possível, mas que apesar do índice relevante é a que foi citada com menor intensidade. Esta dimensão de $\mathrm{OE}$, sugerida e detalhada por Lumpkin e Dess (1996, 2001), indica tendência a responder com vigor às ameaças da concorrência, estando ligada à intensidade com que a organização luta por sua sobrevivência. Entre as características desta dimensão estão o uso de estratégias ousadas, como abrir mão de lucratividade para ampliar participação no mercado. Estes resultados complementam os achados de Lizote et al. (2020), os quais apontam uma postura conservadora no que diz respeito à orientação empreendedora de hotéis em sua precificação.

Lizote et al. (2020) também investigaram orientação empreendedora em conjunto com outras variáveis e concluíram que conhecer sobre a forma predominante de orientação por parte de gestores hoteleiros auxilia no processo de tomada de decisões.
Já entre as dimensões de ACAP, os respondentes perceberam uma maior importância da aquisição de conhecimento nas firmas onde atuam, com $80,41 \%$ do total possível. Esta é uma capacidade de absorção potencial, ou seja, que possibilita a receptividade da empresa ao conhecimento externo, e consiste no primeiro passo da absorção do conhecimento. A aquisição envolve os esforços e rotinas para adquirir o conhecimento.

A assimilação, dimensão apontada com menor importância nas organizações pelos entrevistados, com $66,87 \%$ do total possível, consiste nas rotinas e processos que permitem que estas organizações analisem, processem e interpretem a informação obtida externamente. Esta dimensão prevê ações como trocas efetivas de informações e experiências entre os diferentes departamentos, o que sugere uma integração multidisciplinar para encontrar soluções efetivas para as turbulências enfrentadas pelas organizações.

Os resultados dos achados sobre capacidade absortiva são consonantes aqueles apontados no estudo de Rossetto et al. (2019), os quais demonstram a importância da capacidade absortiva para a compreensão de diversos fenômenos organizacionais, principalmente aqueles relacionados aos processos de gestão do conhecimento, aprendizagem, inovação e desempenho. Também corroboram os estudos de Cappellari et al. (2019, p. 2), ressaltando a importância dos comportamentos e habilidades para a implementação do conhecimento absorvido. Em um ambiente turbulento como o de adaptação dos jornais, a capacidade absortiva pode inclusive favorecer a sustentabilidade, conforme apontam os achados de Padilha et al. (2020).

Os respondentes apontaram as dimensões de capacidade realizada, que consistem em transformar e aplicar o conhecimento, de maneira similar, com $70,67 \%$ e $70,51 \%$, respectivamente. Ambas as dimensões refletem a capacidade de a organização explorar o conhecimento e transformá-lo em inovação (ZAHRA; GEORGE, 2002), portanto estão mais relacionadas com a ação efetivamente. Neste mesmo sentido estão as dimensões de $\mathrm{OE}$ inovatividade, assunção de riscos e agressividade competitiva, fortemente ligadas à prática da inovação.

Os resultados encontrados neste estudo corroboram o estudo de Wales, Parida e Patel (2013), 
confirmando que quanto maior a $\mathrm{OE}$, maior a ACAP. O estudo também confirma os achados de Díaz, Guerrero e Peña (2015), os quais veem na orientação empreendedora e na capacidade absortiva duas características internas imprescindíveis para organizações inovadoras. A adaptação é urgente para os jornais, os quais também estão em sua maioria vivendo uma crise ocasionada pelas mudanças contextuais, conforme o estudo Silva França e Lima Rua (2016) também demonstrou. Os autores apontam a orientação empreendedora e a capacidade absortiva como determinantes para o aumento do desempenho dos negócios nos mercados externos, agregando valor ao atual contexto de mudança (SILVA FRANÇA; LIMA RUA, 2016).

A correlação positiva e significativa entre os constructos centrais do estudo, bem como a manutenção de suas dimensões, corrobora os achados já apontados por Hernandez-Perlines (2018), que constatou que o desempenho internacional de empresas familiares é determinado, em grande parte, pela orientação empreendedora e reforçado pela capacidade absortiva. Os resultados da investigação confirmam também Sbissa et al. (2018), os quais afirmaram que a capacidade absortiva influencia positivamente na orientação empreendedora de hotéis, ampliando o setor de aplicação e público respondente.

As incertezas do mercado em constante transformação, especialmente em uma realidade de queda brusca de receita, o que implica também na capacidade de investimento, pode inibir ações ousadas. A capacidade absortiva, ou seja, os investimentos nos processos ligados à aquisição e aproveitamento de conhecimento externo nas organizações se tornam luz para as firmas. É a partir de exemplos, de análises de tendência de consumo e de busca de alternativas até mesmo inspiradas em outros mercados que a empresa poderá vislumbrar oportunidades para si.

\section{CONSIDERAÇÕES FINAIS}

Foi possível constatar que a capacidade absortiva influencia na orientação empreendedora destas empresas. Isto significa que a forma como estes veículos adquirem, assimilam, transformam e aplicam o conhecimento vai impactar diretamente na maneira como estas organizações empreendem, ou seja, criam novos produtos, processos e serviços capazes de trazer vantagem competitiva. A partir deste resultado, compreende-se que os investimentos nos processos de aprendizagem da organização vão afetar a forma como esta empreende, despertando para a necessidade de aprimoramento do aproveitamento do conhecimento externo. $\mathrm{O}$ interesse primeiro deste estudo, entretanto, não é o que o mesmo pode sugerir às organizações em geral, mas sim, contribuir com as organizações que atuam com jornais impressos, as quais passam por momentos de turbulência.

Como limitação deste estudo, considera-se o fato de que o estudo teve como objeto de estudo unicamente os jornais brasileiros, o que não permite que se faça generalizações aplicáveis aos demais tipos de organização. E ainda, vale citar a amostra utilizada, tendo-se em vista que não foram entrevistadas todas as organizações que compõem o universo pesquisado. Outro ponto é o fato de que as respostas se deram, na grande maioria dos casos, a partir da opinião de apenas um representante da firma, o que pode gerar uma visão individualizada da organização. Além disso, o estudo apresenta uma visão genérica, podendo ser operacionaliza em cada contexto de forma singular, e é guiado por uma visão epistemológica racionalista e positivista do fenômeno.

As limitações oportunizam a sugestão de estudos futuros. Neste sentido, sugere-se a investigação dos mesmos constructos em outros contextos e com uma amostra ampliada. Sugere-se ainda que este estudo seja aplicado em outros segmentos, inclusive no âmbito da indústria da comunicação, ou até mesmo dentro do universo estudado, mas em outros países. Assim, será possível realizar análises para verificar como diferentes variáveis podem influenciar os resultados. Para que este estudo possa ser aprofundado e desta forma contribuir de maneira ainda mais efetiva para o mercado, trabalhos futuros poderiam se utilizar de uma abordagem qualitativa para entender como cada uma das dimensões dos construtos se manifesta nestas organizações. 


\section{REFERÊNCIAS}

ALCADIPANI, R. O declínio dos jornais. GV Executivo, v. 6, n. 2. 2007.

ASSOCIAÇÃO NACIONAL DE JORNAIS (ANJ). A Indústria Jornalística Brasileira em 2017.Site insitucional da ANJ, 2018. Disponível em: <https:// www.anj.org.br/site/servicos/menindjornalistica/ 114-cenarios/742-a-industria-jornalistica-brasileira-em-2017.html>. Acesso em: 28 de abril de 2018.

CAMOZZATO, E. S.; LIZOTE, S. A.; VERDINELLI, M. A.; SERAFIM, F. K. Orientação empreendedora, autoeficácia dos gestores e satisfação com o desempenho: um estudo em empresas incubadas. Revista de Ciências da Administração, Florianópolis, p. 68-83, ago. 2017.

CAPPELLARI, G.; WELTER, C . V. N.; HERMES, L. C. R.; SAUSEN, J. O. Capacidade absortiva: elementos componentes e mecanismos organizacionais de seu desenvolvimento RAM, Rev. Adm. Mackenzie, v. 20, n. 6, eRAMD190028, 2019.

CASSOL, A. Capital intelectual e capacidade absortiva como propulsores da inovação: estudo de caso no setor de papel e papelão ondulado. Dissertação (mestrado) - Programa de Pós-Graduação em Administração, Universidade do Vale do Itajaí, Biguaçu, 2014.

CESTINO, J.; MATTHEWS, R. A perspective on path dependence processes: the role of knowledge integration in business model persistence dynamics in the provincial press in England. Journal of Media Business Studies, v. 13, n. 1, p. 22-44, 2016.

CHURCHILL JR., G. A. A Paradigm for developing better measures of marketing constructs. Journal of Marketing Research, v. 16, n. 1, p. 64-74, 1979.

COHEN, W. M.; LEVINTHAL, D. A. Absorptive Capacity: a new perspective on learning and innovation. Administrative Science Quarterly, v. 35, n. 1, p. 128-152, mar. 1990.
COVIN, J. G.; LUMPKIN, G. T. Entrepreneurial orientation theory and research: Reflections on a needed construct. Entrepreneurship Theory and Practice, v. 35, n. 5, p. 855-872, 2011.

COVIN, J. G.; SLEVIN, D. P. Strategic management of small firms in hostile and benign environments. Strategic management journal, v. 10, n. 1, p. 75-87, 1989.

COVIN, J. G.; WALES, W. J. The measurement of entrepreneurial orientation. Entrepreneurship theory and practice, v. 36, n. 4, p. 677-702, 2012.

DE CLERCQ, D.; DIMOV, D.; THONGPAPANL, N. T. Organizational social capital, formalization, and internal knowledge sharing in entrepreneurial orientation formation. Entrepreneurship Theory and Practice, v. 37, n. 3, p. 505-537, 2013.

DESS, G. G.; LUMPKIN, G. T.. The role of entrepreneurial orientation in stimulating effective corporate entrepreneurship. The Academy of Management Executive, v. 19, n. 1, p. 147-156, 2005.

DÍAZ, Y.; GUERRERO, M.; PEÑA, I. Productividad de la innovación a través del emprendimiento corporativo: Innovation driven productivity through corporate entrepreneurship. Universia Business Review. v. 47, n. 32-47, Jul. 2015. ISSN: 16985117.

DÍAZ-NOCI, J. A History of Journalism on the Internet: A state of the art and some methodological trends. Revista internacional de Historia de la Comunicación, n. 1, p. 253-272, 2013.

ENGELEN, A.; KUBE, H.; SCHMIDT, S.; FLATTEN, T. C. Entrepreneurial orientation in turbulent environments: The moderating role of absorptive capacity. Research Policy, v. 43, n.8, p. 1353-1369, 2014. 
FINNEY, S. J.; DISTEFANO, C. (2013). Nonnormal and categorical data in structural equation modeling. In: HANCOK, G. R.; MUELEER, R. O. Quantitative methods in education and the behavioral sciences: Issues, research, and teaching. Structural equation modeling: a second course (p. 439-492). Charlotte, NC, US: IAP Information Age Publishing.

FRANÇA, A. B.; SARAIVA, J.; HASHIMOTO, M. Orientação empreendedora como indicador do grau de empreendedorismo corporativo: fatores que caracterizam os intraempreendedores e influenciam sua percepção. Revista de Empreendedorismo e Gestão de Pequenas Empresas, v.1, n. 3, 2012.

FREITAS, H.; MARTENS, C. D. P.; BOISSIN, J.; BEHR, A. (2012). Elementos para guiar ações visando à orientação empreendedora em organizações de software. Revista de Administração, v. 47, n. 2, p. 163-179, 2012.

GARCIA, S. D. B.; MARTINEZ, T. L. Análisis de ecuaciones estructurales. In: GREIMEL-FUHRMANN, B.; GEYGER, A. Student's evaluation of teachers and instructional quality analysis of relevant factors based on empirical evaluation research. Assessment Evaluation in Higher Education, London, v. 28, n. 3, p. 229-238, 2003.

GREER, J.; MENSING, D. The evolution of online newspapers: A longitudinal content analysis, 19972003. Internet newspapers: The making of a mainstream medium, p. 13-32, 2006.

GUTH, W. D.; GINSBERG, A.Guest editor's introduction: corporate entrepreneurship. Strategic Management Journal. v. 11, p. 5-15, 1990.

HAIR Jr., J. F.; BLACK, W. C.; BABIN, B. J.; ANDERSON, R. E.; TATHAM R. L.; Análise multivariada de dados. 5. ed. Porto Alegre: Bookman, 2009.
HERNANDEZ-PERLINES, F. Moderating effect of absorptive capacity on the entrepreneurial orientation of international performance of family businesses. Journal of Family Business Management, v. 8, n. 1, p. 58-74, 2018.

HURLEY, R.; HULT, T. Innovation, Market Orientation, and Organizational Learning: an integration and empirical examination. Journal of Marketing, v. 62, n. 3, p. 42-55, 1998.

JAWORSKI, B. J.; KOHLI, A. Market Orientation: antecedents and consequences. Journal of Marketing, v. 57, n. 3, p. 53-70, 1993.

KARIMI, J.; WALTER, Z. Corporate entrepreneurship, disruptive business model innovation adoption, and its performance: The case of the newspaper industry. Long Range Planning, v. 49, n. 3, p. 342-360, 2016.

KIRZNER, I. M. Competition and entrepreneurship. Chicago, IL: Chicago University Press, 1973.

LANE, P. J.; KOKA, B. R.; PATHAK, S. The reification of absorptive capacity: A critical review and rejuvenation of the construct. Academy of management review, v. 31, n. 4, p. 833-863, 2006.

LIZOTE, S. A.; TESTON, S. F.; ZAWADZKI, P., GUPTA, S. K. Entrepreneurial orientation and cost practice in the brazilian south hotel chain. RACE, v. 19, n. 1. p. 19-28, 2020.

LUMPKIN, G. T.; DESS, G. G. Clarifying the entrepreneurial orientation construct and linking it to performance. Academy of management Review, v. 21, n. 1, p. 135-172, 1996.

LUMPKIN, G. T.; DESS, G. G. Linking two dimensions of entrepreneurial orientation to firm performance: The moderating role of environment and industry life cycle. Journal of Business Venturing, v. 16, p. 429-451, 2001. 
MARTENS, Cristina Dai Prá et al. Relação entre Orientação Empreendedora e Maturidade na Gestão De Projetos Em Empresas Brasileiras De Software. 3. ed. [s. L.]: Revista Ibero-americana de Estratégia (RIAE), 2015. 91 p. 14 v.

MARTENS, C. D. P.; LACERDA, F. M.; BELFORT, A. C.; FREITAS, H. M. R. de. Research on entrepreneurial orientation: current status and future agenda. International Journal of Entrepreneurial Behavior \& Research, v. 22, n. 4, p. 556-583, 2016.

MILLER, D. The correlates of entrepreneurship in tree types of firms. Management Science, v. 29, n.7, p. 770-791, 1983.

MILLER, D. Miller (1983) revisited: A reflection on EO research and some suggestions for the future. Entrepreneurship Theory and Practice, v. 35, n. 5, p. 873-894, 2011.

MILLER, D.; FRIESEN, P. Innovation in Conservative and Entrepreneurial Firms: Two Models of Strategic Momentum. Strategic Management Journal, v. 3, p. 1-25, 1982.

MINTZBERG, H. Strategy-Making in Three Modes. California Management Review, v. 16,n. 2,1973.

PADILHA, A. C. M.; CERVO, B.; AZEVEDO, J. B.; VASCONCELOS NETO, A. G.; FAGUNDES, P. M. Capacidade absortiva na produção artesanal de cervejas. Espacios, v. 37, n. 35, 2016.

PADILHA, L. S.; PIEKAS, A. A. S.; KUZMA, E. L.; BEGNINI, S.; CARVALHO, C. E. The impact of the environmental dimensions and the relationship between absorptive capacity and sustainability practices. International Journal of Development Research, v. 10, p. 39985-39991, 2020.

ROSSETO, C. R.; CARVALHO, C. E.; FERREIRA, G. C.; PERY, C. D. Absorptive capacity: the role of external knowledge in organizational strategy. RAM. Revista de Administração Mackenzie (online), v. 20, p. 1, 2019.
SBISSA, A. P. Relacionamento Entre Capacidade Absortiva, Orientação Empreendedora E Desempenho Empresarial: Um Estudo Noshotéisde Florianópolis(SC). Dissertação (Mestrado)Universidade do Vale do Itajaí,Programa de PósGraduação em Administração, Biguaçu, 2017.

SBISSA, A. P.; ROSSETTO, C. R.; CARVALHO, C. E.; ZONATTO, P. A. F. Relacionamento entre capacidade absortiva e orientação empreendedora: um estudo nos hotéis de Florianópolis (SC). Turismo: Visão e Ação, v. 20, p. 311, 2018.

SCHUMPETER, J. The theory of Economic Development:An Inquiry into Profits, Capital, Credit, Interest, and the Business Cycle. Cambride: Harvard University Press, 1934.

SCIASCIA, S.; D’ORIA, L.; BRUNI, M.; LARRAÑETA, B. Entrepreneurial Orientation in low-and medium-tech industries: The need for Absorptive Capacity to increase performance. European Management Journal, v. 32, n. 5, p. 761-769, 2014.

SHANE, S.; VENKATARAMAN, S. The Promise of Entrepreneurship as a Field of Research. The Academy of Management Review, v. 25, n. 1, p. 217-227, 2000.

SLATER, S.; NARVER, J. C. Market orientation and the learning organization. Journal of Marketing, v. 59, n. 3, p. 63-75, 1995.

SILVA FRANÇA, A; LIMA RUA, O. Influence of entrepreneurial orientation and absorptive capacities in export performance. : Influência da orientação empreendedora e das capacidades abortivas no desempenho das exportações. Tourism \& Management Studies. v. 12, n. 1, p. 196-202, Jan. 2016. ISSN: 16462408.

TEECE, D. J. Explicating dynamic capabilities: Asset selection, coordination, and entrepreneurship in strategic management theory. UC Berkeley, 2003. 
TEECE, D. J.; PISANO, G.; SHUEN, A. Dynamic capabilities and strategic management. Strategic management journal, p. 509-533, 1997.

TENCONI, C. D. Desenvolvimento de uma Escala para mensurar a capacidade de absorção em pequenas empresas. 2015. 251 f. Dissertação (Mestrado) -Curso de Administração, Universidade Estadual de Santa Catarina, Florianópolis, 2015.

TONDOLO, V. A. G.; BITENCOURT, C. C. Compreendendo as capacidades dinâmicas a partir de seus antecedentes, processos e resultados. Brazilian Business Review, v. 11, n. 5, p. 124-147, 2014.

VAN DOORN, S.; HEYDEN, M. L. M; VOLBERDA, H. W. Enhancing Entrepreneurial Orientation in Dynamic Environments: The Interplay between Top Management Team Advice-Seeking and Absorptive Capacity. Long Range Planning, v. 50, n. 2, p. 134144, 2017.

WALES, W. J.; PARIDA, V.; PATEL, P. C. Too much of a good thing? Absorptive capacity, firm performance, and the moderating role of entrepreneurial orientation. Strategic Management Journal, v. 34, n. 5, p. 622-633, 2013.

WANG, C. L.; AHMED, P. K. Dynamic capabilities: A review and research agenda. International journal of management reviews, v. 9, n. 1, p. 31-51, 2007.

ZAHRA, S. A.; GEORGE, G. Absorptive capacity: A review, reconceptualization, and extension. Academy of management review, v. 27, n. 2, p. 185-203, 2002. 